\title{
Vortex Pinning in Nb Films Patterned with Nano-scale Hole-arrays
}

\author{
U. Welp ${ }^{\mathrm{a}, 1}$, Z. L. Xiao ${ }^{\text {a }}$, J. S. Jiang ${ }^{\text {a }}$, V. K. Vlasko-Vlasov ${ }^{\text {a }}$, S. D. Bader ${ }^{\text {a }}$, \\ G. W. Crabtree ${ }^{\mathrm{a}}$, J. Liang ${ }^{\mathrm{b}}$, H. Chik ${ }^{\mathrm{b}}$, J. M. Xu ${ }^{\mathrm{b}}$ \\ a Materials Science Division. Argonne National Laboratory, Argonne, IL 60439 \\ ${ }^{\mathrm{b}}$ Division of Engineering and Department of Physics, Brown University, Providence, RI 02912
}

\begin{abstract}
$\mathrm{Nb}$ films containing extended arrays of holes with 45-nm diameter and 100-nm spacing have been fabricated using anodized aluminum oxide (AAO) as substrate. Pronounced matching effects in the magnetization are seen in fields up to $9 \mathrm{kOe}$. Flux pinning in the patterned samples is enhanced by two orders of magnitude as compared to unpatterned reference samples in applied fields exceeding $5 \mathrm{kOe}$. The matching effects are a dominant contribution to vortex pinning at temperatures as low as $4.2 \mathrm{~K}$ due to the nano-scale spacing of the holes.
\end{abstract}

Key words: periodic pinning, superconducting networks

\section{Introduction}

The behavior of superconducting vortices in the presence of periodic arrays of pinning sites has attracted much recent attention because of technological as well as scientific interest. In these systems pronounced commensurability effects [1] including large enhancements of the critical current, dips in the magneto-resistance and in the flux creep rate have been observed at fields corresponding to integer multiples or to simple fractions of the matching field, $H_{1}$. At $H_{1}$ the number of vortices equals the number of pinning sites.

Here we show that anodized aluminum oxide (AAO) membranes are ideal substrates for growing laterally patterned superconducting films. Large area (several $\mathrm{cm}^{2}$ ) Nb films containing triangular lattices of 45-nm diameter holes with 100-nm period have been prepared. These samples, having the smallest feature size in extended patterns yet reported, show pronounced commensurability effects even at liquid He-temperature in

1 Corresponding author. Present address: Materials Science Division, Argonne National Laboratory, 9700 S. Cass Avenue, Argonne, IL 60439 E-mail: welp@anl.gov fields up to $9 \mathrm{kOe}$. Enhancements of the critical current by more than two orders of magnitude in fields of several $\mathrm{kOe}$ have been achieved. The unique feature of these samples is that the zero-temperature coherence length, $\xi(0)$, and penetration depth, $\lambda(0)$, are of the same order as the pattern period ensuring that even at low temperatures the potential energy of the vortices is dominantly periodic.

\section{Results}

A two step process [2] was used to fabricate the AAO membranes. $100 \mathrm{~nm}$ thick $\mathrm{Nb}$-films and $10 \mathrm{~nm}$ thick Ag-cap layers were deposited directly onto the AAO using magnetron sputtering. Simultaneously, continuous reference samples were grown on sapphire substrates. Fig. 1 shows a SEM image of the sample. The holes with average diameter $\mathrm{d}=45 \mathrm{~nm}$ are arranged on a triangular lattice with period $\mathrm{a}=101 \mathrm{~nm}$. Within domains of several $\mu \mathrm{m}$ size the holes are perfectly ordered. $T_{c}$ of the patterned Nb sample is $6.85 \mathrm{~K}$ whereas for the reference sample it is $7.5 \mathrm{~K}$. This reduction in $T_{\mathrm{c}}$ can be caused in part by the proximity with the $\mathrm{Ag}$ cap layer and by contamination and/or interdiffusion 


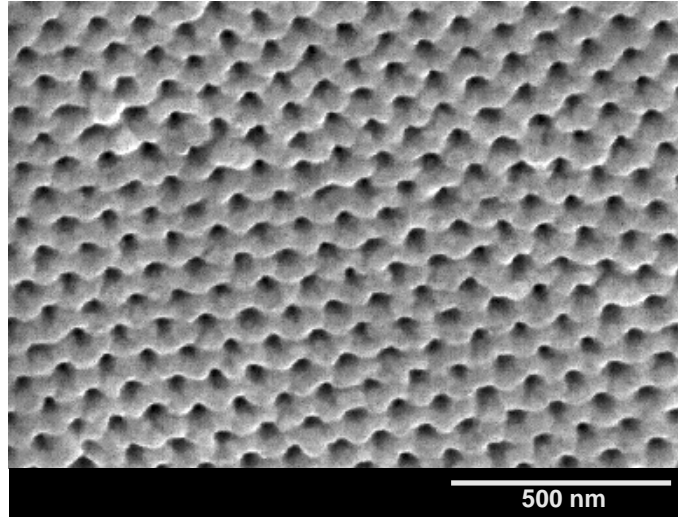

Fig. 1. SEM image of the patterned Nb-film. The holes with diameter of $45 \mathrm{~nm}$ are arranged on a triangular lattice with period $101 \mathrm{~nm}$

at the $\mathrm{AAO} / \mathrm{Nb}$ interface.

Fig. 2 shows the magnetization hysteresis, $\Delta m$, of the patterned sample and of the reference sample at several temperatures. $\Delta m$ of the patterned film is characterized by a sequence of pronounced cusps occuring at multiples of $H_{1}=2300$ Oe. This value is in good agreement with the expected value $2 \Phi_{0} / \sqrt{3} a^{2}=2255$ Oe demonstrating that the observed magnetization features are a result of matching. Due to the large value of $H_{1}$ effects of matching are observed in fields up to $9 \mathrm{kOe}$. In contrast, matching effects in previous studies on $\mathrm{Nb}$-, $\mathrm{Pb}$ - or high- $T_{\mathrm{c}}$-samples have been generally limited to fields below 100 Oe [1] and to temperatures close to $T_{c}$. The nano-scale periodic pinning array induces a significant enhancement of vortex pinning in the patterned sample as compared to the reference sample. Whereas $\Delta m$ of the reference sample drops exponentially to negligible values in 1 to $2 \mathrm{kOe} \Delta m$ of the patterned sample is almost field independent (between the matching fields) for fields up to $5 \mathrm{kOe}$. Thus, a desired field dependence of the critical current can be engineered by introducing a properly designed pin array.

For applied fields $H \leq H_{1}$ pinning and matching are caused by trapping the vortices in the holes. In fields $H_{\mathrm{n}}=\mathrm{n} H_{1}$ with $\mathrm{n}>1$ matching effects can arise either from caging of interstitial vortices between the holes [3] or pinning of multi-quanta vortices in the holes [4]. Doubly quantized vortices in a triangular array of cylindrical cavities are stable [4] for $d^{3}>8 a^{2}$. Measurements of the critical fields of the patterned samples [5] yielded a coherence length and penetration depth of $\xi(0) \sim 10 \mathrm{~nm}$ and $\lambda(0) \sim 70 \mathrm{~nm}$, respectively. Thus, the stability condition is not fulfilled for our samples. Similarly, for an isolated hole it has been shown [6] that the attractive pinning force vanishes if the number of vortices confined to the cavity exceeds the saturation number $n_{s}=d / 4 \xi(T)$, which yields $n_{s}=1$ for our

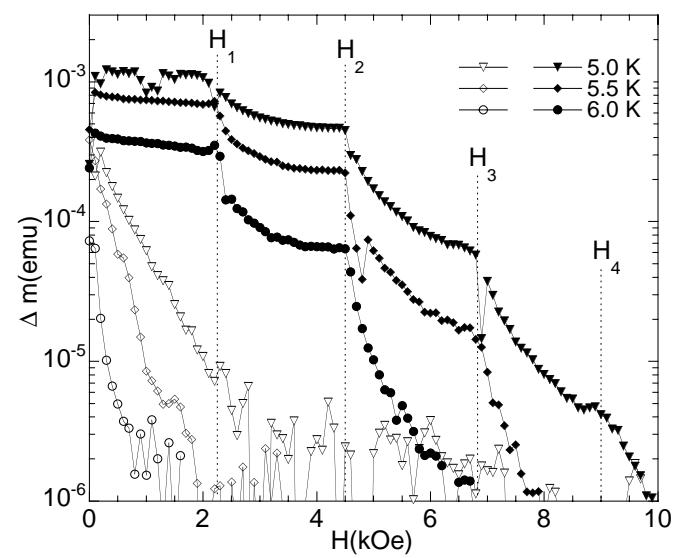

Fig. 2. Magnetization hysteresis at several temperatures of the patterned Nb-film (closed symbos) and of the reference film (open symbols). Both samples are approximately square shaped with $2 \mathrm{~mm}$ sides.

samples. Therefore, the matching features at $H_{\mathrm{n}}, \mathrm{n}$ $>1$, are a manifestation of interstitial vortices being pinned by a confining cage potential exerted by vortices trapped in the holes. This potential is determined by the ratio $\lambda(T) / a$ and the number of interstitials per unit cell. As this ratio decreases, the cage potential is rapidly smoothed out, and the periodic pinning potential for interstitial vortices disappears. In our samples, $\lambda(T) / a \sim 1$ assuring that the periodic caging potential is a dominant contribution to the pinning force over the entire temperature range.

\section{Acknowledgements}

This work was supported by the U.S. Department of Energy, BES, Materials Science under contract W31-109-ENG-38 at Argonne National Laboratory, and by the NSF under contract NSF ECS 0070019 and by the ONR under contract N000140010260 at Brown University.

\section{References}

[1] A. T. Fiory et al., Appl. Phys. Lett. 32 (1978) 73; M. Baert et al., Phys. Rev. Lett. 74 (1995) 3269; A. Castellanos et al., Appl. Phys. Lett. 71 (1997) 962; V. V. Metlushko et al., Phys. Rev. B 59 (1999), 603; ibid. 60 (1999) R12585.

[2] A. J. Yin et al., Appl. Phys. Lett. 79 (2001) 1039; J. Liang et al., J Appl. Phys 91 (2002) 2544; H. Masuda, H. Fukuda, Science 268 (1995) 1466.

[3] I. B. Khalfin, B. Ya. Shapiro, Physica C 207 (1993) 359

[4] A. I. Buzdin, Phys. Rev. 47 (1993) 11416

[5] U. Welp et al., cond-mat 0204535.

[6] G. S. Mkrtchyan, V. V. Schmidt, Soviet Phys. JETP 34 (1972) 195 